\title{
Efficient kinetic Monte Carlo method for reaction-diffusion processes with spatially varying annihilation rates
}

\author{
Karsten Schwarz 1 , 因 and Heiko Rieger ${ }^{1, \Theta}$ \\ ${ }^{1}$ Theoretische Physik, Universität des Saarlandes, 66041 Saarbrücken, Germany
}

(Dated: November 6, 2018)

\begin{abstract}
We present an efficient Monte Carlo method to simulate reaction-diffusion processes with spatially varying particle annihilation or transformation rates as it occurs for instance in the context of motor-driven intracellular transport. Like Green's function reaction dynamics and first-passage time methods, our algorithm avoids small diffusive hops by propagating sufficiently distant particles in large hops to the boundaries of protective domains. Since for spatially varying annihilation or transformation rates the single particle diffusion propagator is not known analytically, we present an algorithm that generates efficiently either particle displacements or annihilations with the correct statistics, as we prove rigorously. The numerical efficiency of the algorithm is demonstrated with an illustrative example.
\end{abstract}

Keywords: computer simulations, kinetic Monte Carlo, reaction-diffusion processes

\section{INTRODUCTION}

Kinetic Monte Carlo simulations are frequently used in various fields to analyze the spatio-temporal evolution of systems consisting of many freely diffusing particles that can collide, react, transform or annihilate. Spatial as well as stochastic aspects are important when diffusion is not sufficiently fast to make the system well-stirred and the number of reactants within diffusion range is small. In this case a mean-field description, for instance with a set of coupled reaction-diffusion equations, is inappropriate. Moreover, in the limit of extreme dilution methods using a discretization of the underlying stochastic reaction-diffusion system, either in time [1] or in space [2, 3], become computationally inefficient.

The currently most efficient methods to simulate extremely diluted reaction-diffusion systems are Green's function reaction dynamics [4, 5] and first-passage kinetic Monte Carlo methods [6 8]. In essence they avoid the small diffusion hops of a conventional random walk or Brownian dynamics simulation by propagating particles over long distances through a sequence of large displacements. The latter are generated stochastically according to the exactly known Green's function for a freely diffusing particle within so-called protective domains that are free from other particles. The typical size of these protective domains is inversely proportional to the particle density and the larger these domains are (i.e. the smaller the particle density is) the more efficient the algorithm is.

In general, during the free diffusion the particle can also be annihilated or transformed with a rate $k$ into a different species, in which case the Green's function is still exactly know. In this paper we address the question how to propagate the particles when the annihilation rate

\footnotetext{
*Electronic address: kschwarz@lusi.uni-sb.de

${ }^{\dagger}$ Electronic address: h.rieger@mx.uni-saarland.de
}

varies in space and time, denoted as $k(\mathbf{r}, t)$. This problem arises for instance in the context of motor-driven intracellular transport, where particles (or cargos) can in addition to diffusion and reaction also attach to a cytoskeleton filament and move ballistically with a constant speed in the direction of the filament. A continuum description of the diffusive and ballistic modes of motion [9, 10] involves the filament density $\rho(\mathbf{r}, t)$ which determines the local rate with which freely diffusing particles make a transition into the ballistic state. In a typical cell the filament density is spatially inhomogeneous and thus has to be taken into account during the propagation of particles on large scales. Analogous examples arise in systems in which the annihilation of particles depends on a spatially inhomogeneous concentration field of an abundant reaction partner (i.e. whose density is much larger such that a continuum description is appropriate for it).

Green's function reaction dynamics and first-passage time Monte Carlo methods reduce the simulation of a many-particle reaction-diffusion system to individual particles that diffuse freely as long as other particles are sufficiently distant (i.e. outside the interaction range), and perform a reaction event once a particle pair reaches a minimum distance. Algorithmically one can ensure free diffusion for instance by estimating the maximum diffusion distance [4, 5] until a reaction is scheduled or by the definition of protective domains for each particle [68] depending on the actual arrangement of neighboring particles. In both cases one then utilizes the free diffusion propagator within predefined domains to generate stochastically a time when either the maximum distance is underrun or a protective domain boundary is reached. For free diffusion this is achieved using the analytically known Green's function, but for free diffusion with spatially varying annihilation rates this propagator is unfortunately not analytically available.

Thus in this paper we consider a freely diffusing single particle in an arbitrary domain $G \in R^{n}$ that can be annihilated with a time and space dependent rate $k(\mathbf{r}, t)$. 
In general, annihilation means a transition into a different species that is not considered in the present reduced setup. For a particle initially at time $t_{0}$ located at $\mathbf{r}_{\mathbf{0}} \in G$ this diffusion-annihilation process is described by the following diffusion-annihilation equation

$\frac{\partial P\left(\mathbf{r}, t \mid \mathbf{r}_{0}, t_{0}\right)}{\partial t}=D \Delta P\left(\mathbf{r}, t \mid \mathbf{r}_{0}, t_{0}\right)-k(\mathbf{r}, t) P\left(\mathbf{r}, t \mid \mathbf{r}_{0}, t_{0}\right)$,

where $P\left(\mathbf{r}, t \mid \mathbf{r}_{0}, t_{0}\right)$ is the probability density to find the particle at time $t$ at $\mathbf{r} \in G$. For arbitrary $k(\mathbf{r}, t)$ and arbitrary $G$ there is no analytic solution of Eq. (1) available. In principle this equation can be solved numerically, but in the context of a general reaction-diffusion system (involving many particles and several particle species) using for instance the first-passage Monte Carlo method this is unfeasible: Here one needs for each particle hop the whole first-passage time distribution for a particle to reach the protective domain boundary $\partial G$, which is computationally too demanding to be carried out in the innermost loop of the algorithm.

Therefore we present in this paper an algorithm that samples times $t>t_{0}$ and positions $\mathbf{r}$ for arbitrary annihilation rates $k(\mathbf{r}, t)$ and arbitrary domains for which a particle diffusing according to Eq. (10) either a) reaches the boundary for the first time $(\mathbf{r} \in \partial G)$ or b) is annihilated $(\mathbf{r} \in G)$. In addition, a slightly modified version of the algorithm generates the whole probability density $P\left(\mathbf{r}, t \mid \mathbf{r}_{0}, t_{0}\right)$ within $G$, meaning it solves Eq. (1) stochastically.

The paper is organized as follows: Section 1 defines all probability densities and flows used throughout this paper. Based on the ideas of [4 [8], section Џ1Ipresents an algorithm for the sampling of $(\mathbf{r}, t)$ on arbitrary domains $G$ in the case of a spatially homogeneous but temporally varying annihilation rate $k(\mathbf{r}, t)=k(t)$. Section IV generalizes this method to spatially inhomogeneous rates $k(\mathbf{r}, t)$, proves its correctness and discusses its efficiency. Finally section $\nabla$ shows an application example of this method.

\section{DEFINITIONS}

In this section the probability densities and flows used later on are defined. Let $P\left(\mathbf{r}, t \mid \mathbf{r}_{0}, t_{0}\right)$ be the probability density solving the diffusion-annihilation equation (1) within the domain $G$ with boundary $\partial G$, possibly partly absorbing, partly reflecting. The particle annihilation generates a probability flow $f_{a}\left(\mathbf{r}, t \mid \mathbf{r}_{0}, t_{0}\right)$ out of the system given by

$$
f_{a}\left(\mathbf{r}, t \mid \mathbf{r}_{0}, t_{0}\right)=k(\mathbf{r}, t) \cdot P\left(\mathbf{r}, t \mid \mathbf{r}_{0}, t_{0}\right) .
$$

The probability flow $f_{b}\left(\mathbf{r}, t \mid \mathbf{r}_{0}, t_{0}\right)$ at the absorbing parts of the boundary at time $t$ at position $\mathbf{r} \in \partial G$ is given by

$$
f_{b}\left(\mathbf{r}, t \mid \mathbf{r}_{0}, t_{0}\right)=-D \nabla P\left(\mathbf{r}, t \mid \mathbf{r}_{0}, t_{0}\right) \cdot \mathbf{n}_{\mathbf{r}},
$$

where $\mathbf{n}_{\mathbf{r}}$ denotes the outward pointing unity vector perpendicular to the boundary $\partial G$ at $\mathbf{r}$. Consequently $P\left(\mathbf{r}, t \mid \mathbf{r}_{0}, t_{0}\right)$ is not normalized for $t>t_{0}$. The corresponding probability density $\rho_{e}\left(t \mid \mathbf{r}_{0}, t_{0}\right)$ for an annihilation or absorption event is given by

$$
\begin{aligned}
& \rho_{e}\left(t \mid \mathbf{r}_{0}, t_{0}\right)=-\frac{d}{d t}\left[\int_{G} d \mathbf{r} P\left(\mathbf{r}, t \mid \mathbf{r}_{0}, t_{0}\right)\right] \\
& =\alpha\left(t \mid \mathbf{r}_{0}, t_{0}\right)+\beta\left(t \mid \mathbf{r}_{0}, t_{0}\right) \\
& \text { with } \quad \alpha\left(t \mid \mathbf{r}_{0}, t_{0}\right)=\int_{G} d \mathbf{r} f_{a}\left(\mathbf{r}, t \mid \mathbf{r}_{0}, t_{0}\right) \\
& \text { and } \beta\left(t \mid \mathbf{r}_{0}, t_{0}\right)=\int_{\partial G} d F f_{b}\left(\mathbf{r}, t \mid \mathbf{r}_{0}, t_{0}\right),
\end{aligned}
$$

where $d F$ denotes the surface element at position $\mathbf{r} \in \partial G$. Hence, the task is to sample the pairs $(\mathbf{r}, t)$ in statistical agreement to $f_{a}\left(\mathbf{r}, t \mid \mathbf{r}_{0}, t_{0}\right)$ and $f_{b}\left(\mathbf{r}, t \mid \mathbf{r}_{0}, t_{0}\right)$, i.e the statistic of $t$ will be according to $\rho_{e}$.

In the following we also need the probability distribution of a freely diffusing particle $P_{D}\left(\mathbf{r}, t \mid \mathbf{r}_{0}, t_{0}\right)$ without annihilation, which obeys

$$
\frac{\partial P_{D}\left(\mathbf{r}, t \mid \mathbf{r}_{0}, t_{0}\right)}{\partial t}=D \Delta P_{D}\left(\mathbf{r}, t \mid \mathbf{r}_{0}, t_{0}\right) .
$$

The probability density for being absorbed at the boundary for a purely diffusing particle at time $t$ is given by

$$
\rho_{b}^{D}\left(t \mid \mathbf{r}_{\mathbf{0}}, t_{0}\right)=-\frac{d}{d t} \int_{G} d \mathbf{r} P_{D}\left(\mathbf{r}, t \mid \mathbf{r}_{0}, t_{0}\right)
$$

and the probability density of the absorbing position $\mathbf{r} \in$ $\partial G$ under the condition that the absorption takes place at time $\mathrm{t}$ is given by

$$
\rho_{f}^{D}\left(\mathbf{r} \mid t, \mathbf{r}_{0}, t_{0}\right)=\frac{\nabla P_{D}\left(\mathbf{r}, t \mid \mathbf{r}_{0}, t_{0}\right) \cdot \mathbf{n}_{\mathbf{r}}}{\int_{\partial G} d F \nabla P_{D}\left(\mathbf{r}, t \mid \mathbf{r}_{0}, t_{0}\right) \cdot \mathbf{n}_{\mathbf{r}}} .
$$

Using the Gauss's theorem and Eq. (5D) in the denominator, one obtains:

$$
\rho_{f}^{D}\left(\mathbf{r} \mid t, \mathbf{r}_{0}, t_{0}\right)=-D \frac{\nabla P_{D}\left(\mathbf{r}, t \mid \mathbf{r}_{0}, t_{0}\right) \cdot \mathbf{n}_{\mathbf{r}}}{\rho_{b}^{D}\left(t \mid \mathbf{r}_{\mathbf{0}}, t_{0}\right)} .
$$

For spatially homogeneous annihilation rates $k(\mathbf{r}, t)=$ $k(t)$ the annihilation process decouples from all spatial variables, i.e. the solution of Eq. (1) can be written as

$$
P\left(\mathbf{r}, t \mid \mathbf{r}_{0}, t_{0}\right)=e^{-\int_{t_{0}}^{t} k\left(t^{\prime}\right) d t^{\prime}} \cdot P_{D}\left(\mathbf{r}, t \mid \mathbf{r}_{0}, t_{0}\right) .
$$

Hence, the probability density of being annihilated at time $t$ under the condition of not being absorbed at the 
boundary before for a spatially homogeneous rate $k(t)$ is given by

$$
\rho_{a}^{D}\left(t \mid t_{0}\right)=-\frac{d}{d t}\left[e^{-\int_{t_{0}}^{t} k\left(t^{\prime}\right) d t^{\prime}}\right]
$$

and the probability density of the annihilation position $\mathbf{r} \in G$ under the condition that the particle is annihilated at time $t$ is given by

$$
\rho_{n}^{D}\left(\mathbf{r} \mid t, \mathbf{r}_{\mathbf{0}}, t_{0}\right)=\frac{P_{D}\left(\mathbf{r}, t \mid \mathbf{r}_{0}, t_{0}\right)}{\int_{G} d \mathbf{r}^{\prime} P_{D}\left(\mathbf{r}^{\prime}, t \mid \mathbf{r}_{0}, t_{0}\right)},
$$

which is equal to the probability density of a purely diffusing particle under the condition of not being absorbed.

\section{HOMOGENEOUS ANNIHILATION RATE}

In this section we present an algorithm that samples times $t>t_{0}$ and positions $\mathbf{r}$ for homogeneous annihilation rates $k(\mathbf{r}, t)=k(t)$ and arbitrary domains $G$ for which a particle diffusing according to Eq. (1D) either a) reaches the boundary for the first time $(\mathbf{r} \in \partial G)$ or b) is annihilated $(\mathbf{r} \in G)$.

Assuming the ability to generate random numbers according to all the densities, which were defined in the previous section, a correct way of sampling $(\mathbf{r}, t)$ is shown in Algorithm 1

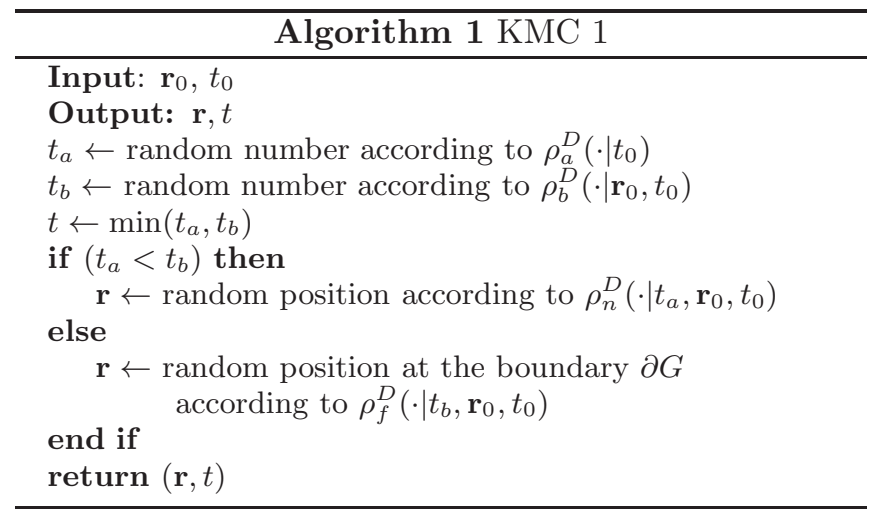

The probability density $A(\mathbf{r}, t)$ that the algorithm produces an annihilation at time $t$ at position $\mathbf{r}$ is then given by

$$
\begin{aligned}
A(\mathbf{r}, t) & =\rho_{a}^{D}\left(t \mid t_{0}\right)\left(\int_{t}^{\infty} d t_{b} \rho_{b}^{D}\left(t_{b} \mid \mathbf{r}_{0}, t_{0}\right)\right) \rho_{n}^{D}\left(\mathbf{r} \mid t, \mathbf{r}_{0}, t_{0}\right) \\
& =\rho_{a}^{D}\left(t \mid t_{0}\right) P_{D}\left(\mathbf{r}, t \mid \mathbf{r}_{0}, t_{0}\right) \\
& =f_{a}\left(\mathbf{r}, t \mid \mathbf{r}_{0}, t_{0}\right) .
\end{aligned}
$$

The probability density $B(\mathbf{r}, t)$ that the algorithm delivers an absorption at time $t$ at position $\mathbf{r} \in \partial G$ is given by

$$
\begin{aligned}
B(\mathbf{r}, t) & =\left(\int_{t}^{\infty} d t_{a} \rho_{a}^{D}\left(t_{a} \mid t_{0}\right)\right) \rho_{b}^{D}\left(t \mid \mathbf{r}_{0}, t_{0}\right) \rho_{f}^{D}\left(\mathbf{r} \mid t, \mathbf{r}_{\mathbf{0}}, t_{0}\right) \\
& =f_{b}\left(\mathbf{r}, t \mid \mathbf{r}_{0}, t_{0}\right)
\end{aligned}
$$

Consequently, the statistic of random pairs $(\mathbf{r}, t)$ generated in this way coincides with $f_{a}\left(\mathbf{r}, t \mid \mathbf{r}_{0}, t_{0}\right)$ and $f_{b}\left(\mathbf{r}, t \mid \mathbf{r}_{0}, t_{0}\right)$ and is therefore correct.

One problem remains: As there are no analytic solutions for Eq. (5) available for arbitrary domains $G$, it is not possible to sample the quantities $\rho_{f}^{D}, \rho_{n}^{D}$ and $\rho_{b}^{D}$ directly for arbitrary domains $G$ and arbitrary boundary conditions. Only a direct sampling of $\rho_{a}^{D}$ is possible, as there is always an analytic expression for the corresponding distribution function available:

$$
F_{a}^{D}\left(t \mid t_{0}\right)=1-e^{-\int_{t_{0}}^{t} k\left(t^{\prime}\right) d t^{\prime}} .
$$

That means, Algorithm 1 is useful only in some special geometries $G$.

Nevertheless, it is possible to use these special cases to sample the random pair $(\mathbf{r}, t)$ for arbitrary domains. Two different methods will be shown now.

\section{A. Subset method}

In [6 8], a kinetic Monte Carlo method for the simulation of reaction-diffusion processes of many-body systems is presented. It is based on the fact that there are analytic solutions of Eq. (5) for some simple domains $G^{\prime}$ and boundary conditions. The appendix shows a list with some of these domains in one, two and three dimensions and derives expressions for distribution functions, which are necessary for the usage of the inversion method [11].

If $G^{\prime}$ denotes a subset of $G$ with $\mathbf{r}_{0} \in G^{\prime}$, the shape of $G \backslash G^{\prime}$ will not matter for the particle, as long as the particle has not left $G^{\prime}$ for the first time. Hence, we can restrict the description of the particle's motion to $G^{\prime}$ until it leaves $G^{\prime}$ for the first time. Mathematically we are dealing with a first-passage-problem in $G^{\prime}$. Its solution is given by Eq. (5) on $G^{\prime}$ according to absorbing boundary conditions at the interior of $G$ and the boundary conditions of $G$ at common boundaries of $G$ and $G^{\prime}$ (as far as they exist).

Assuming that we are able to sample all occurring densities, a random pair $(\mathbf{r}, t)$ for $G^{\prime}$ can be generated, as shown in Algorithm [1. If annihilation takes place $\left(t_{a}<t_{b}\right)$, the particle is annihilated before it leaves $G^{\prime}$ and therefore it is not influenced by the restriction to $G^{\prime}$. If the particle reaches the boundary of $G^{\prime}\left(t_{b}<t_{a}\right)$, two possibilities have to be distinguished: For $\mathbf{r} \in \partial G$ it reaches an absorbing boundary of $G$ and the algorithm will stop. Otherwise, the particle continues its diffusive motion under the condition of having been at position $\mathbf{r}_{0}=\mathbf{r}$ at time $t_{0}=t$. As it is always possible (see appendix) for an arbitrary $\mathbf{r}_{0}$ to find a subset of $G$ where there are all needed analytic expressions available, we can go on this way until the particle is annihilated or absorbed at the boundary of $G$. The pseudo-code of this is shown in Algorithm 2 . 

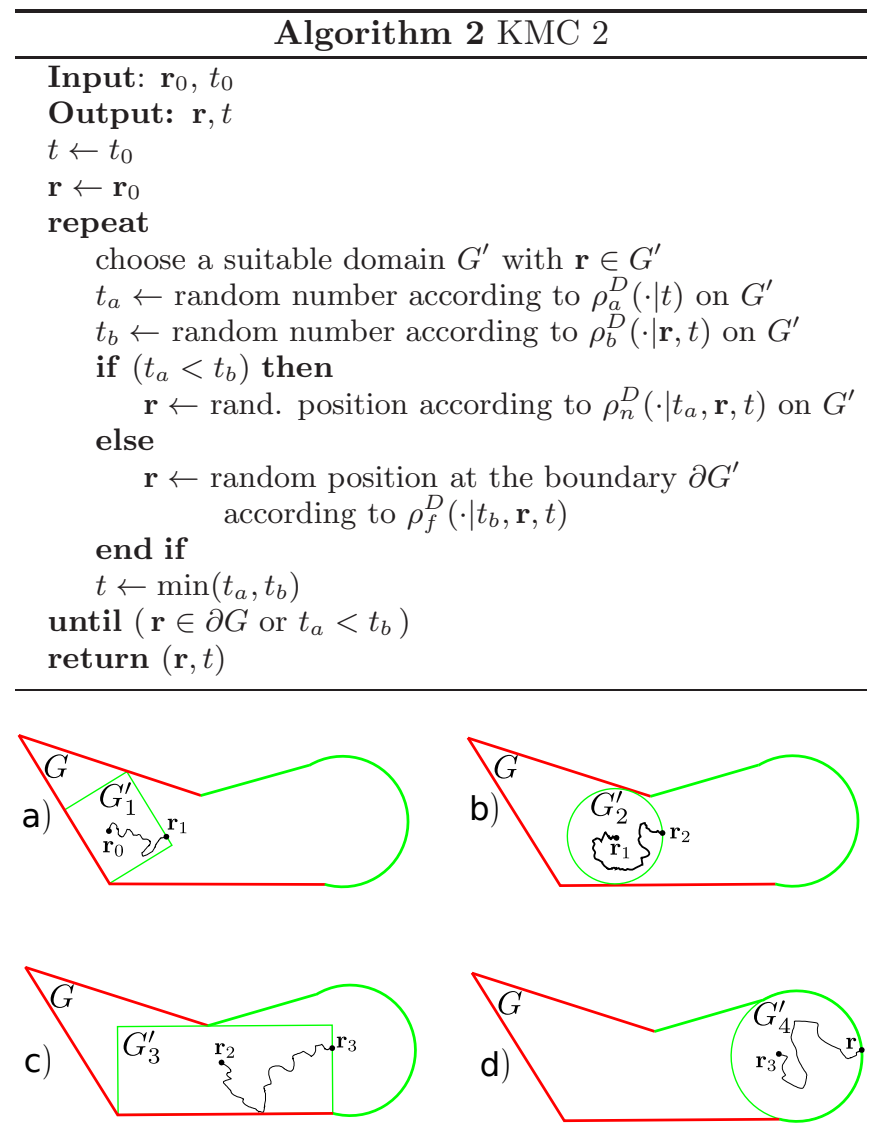

FIG. 1: Illustration of the method: Absorbing boundaries are shown in green, reflecting ones in red. In all situations a)-d) the case $t_{a}>t_{b}$ is sketched, otherwise the algorithm would stop earlier. Depending on the position of the particle the shape of the chosen domain $G^{\prime}$ varies (rectangles and circles).

A sketch of the method in a case where the particle is absorbed at the boundary is shown in Fig. 1.

For a given domain $G$, the efficiency of the method depends on the choices of $G^{\prime}$. Ideally, one chooses $G^{\prime}$ from the list of possibilities in a way that maximizes the expectation value of $t_{b}$. However, it also takes more time to look for this special subset and eventually calculate the random numbers for this situation. In the cases of a particle in the middle of a circle (sphere) or in the middle of a square (cube) the random numbers can be generated very fast. Hence, in some situations it might be better to use smaller domains $G^{\prime}$ than in principle possible.

Up to this point, there is no approximation involved, but depending on the shape of $\partial G$ a problem occurs: If the particle approaches an absorbing part of the boundary of $G$, it will always automatically approach an absorbing part of the boundary of the chosen $G^{\prime}$, too, as $G^{\prime} \subset G$. In consequence, the expectation value of $t_{b}$ will decrease, the stopping condition $t_{a}<t_{b}$ becomes more and more unlikely and the time incrementations in $t$ will become smaller and smaller, if the condition $\mathbf{r} \in \partial G$ is not fulfilled. But $\mathbf{r} \in \partial G$ can only be true, if the intersection of $\partial G$ and $\partial G^{\prime}$ contains more than just single points.
The same problem occurs for reflecting boundaries of $G$. As the choice of $G^{\prime}$ is limited, we sometimes have to approximate $\partial G$ by a polygon in order to avoid a critical slowing down of the algorithm. However, it is important to mention that we can always choose the accuracy of the approximation by the choice of the polygon.

\section{B. Maximum distance method}

Depending on the shape of $\partial G$ close to the position of the particle, there is sometimes a better way of propagating the particle than it is shown in the subsection above. [4, 5] introduced this idea for the particle's short time behavior in the context of particle-particle interaction, but it can be modified for a usage in our context. It is based on the assumption that there is a maximum distance $\Delta r$, which the particle does not reach within a time $\Delta t$. Hence, within this time interval $\Delta t$, only the intersection of $G$ with a neighborhood of radius $\Delta r$ matters. Of course, this assumption is an approximation since there is a non-vanishing probability that the particle leaves this neighborhood within $\Delta t$. However, it is possible to control the accuracy by the definition of $\Delta r$ via a parameter $\gamma$. We define:

$$
\Delta r=\gamma \sqrt{D \Delta t}
$$

As $\gamma$ increases, it is more and more unlikely for the particle to violate the assumption. More precisely it is even possible to give an upper boundary for failing the assumption by studying the first passage-process to the boundary of a particle that starts in the middle of a circle (2d) or a sphere (3d) with radius $\Delta r$ and calculating the probability $w(\gamma)$ for not having reached the boundary within $\Delta t$.

In 2 dimensions we obtain:

$$
w_{2 d}(\gamma)=2 \sum_{n=1}^{\infty} \frac{1}{\alpha_{n}} \frac{1}{J_{1}\left(\alpha_{n}\right)} e^{-\frac{\alpha_{n}^{2}}{\gamma^{2}}}
$$

where $\alpha_{n}, n \in \mathbb{N}$ are the roots of the Bessel function $J_{0}$ (see appendix and be aware of the slightly different notation). In the following tabular the corresponding values are calculated for some $\gamma$.

\begin{tabular}{|r||r|r|r|r|r|r|}
\hline$\gamma$ & 2 & 3 & 4 & 6 & 7 & 9 \\
\hline $1-w_{2 d}(\gamma)$ & 0.623 & 0.193 & 0.0347 & $2.41 \mathrm{e}-4$ & $9.39 \mathrm{e}-06$ & $3.90 \mathrm{e}-9$ \\
\hline
\end{tabular}

In 3 dimensions we get (see appendix):

$$
w_{3 d}(\gamma)=2 \sum_{n=1}^{\infty}(-1)^{n+1} e^{-\left(\frac{n \pi}{\gamma}\right)^{2}}
$$

In the following tabular the corresponding values are calculated for some $\gamma$. 


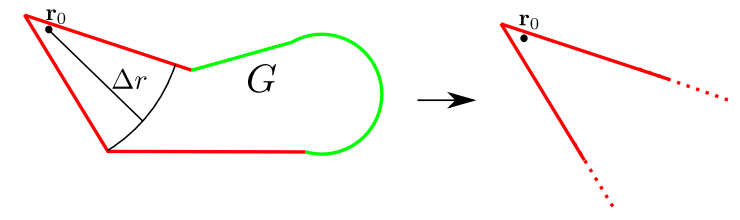

FIG. 2: For $t<\Delta r^{2} / \gamma^{2} D$, the particle is assumed not to cross the black circle line. So the solution of an infinite sector with reflecting boundaries can be used.

\begin{tabular}{|r||r|r|r|r|r|r|}
\hline$\gamma$ & 2 & 3 & 4 & 6 & 7 & 9 \\
\hline $1-w_{3 d}(\gamma)$ & 0.830 & 0.357 & 0.0827 & $8.36 \mathrm{e}-4$ & $3.78 \mathrm{e}-05$ & $1.63 \mathrm{e}-8$ \\
\hline
\end{tabular}

Consequently, for a choice of $\gamma$ in the range of $7-9$ one is on the safe side for all practical purposes, where also other numerical error sources (quality of the random number generator, rounding errors) come into play.

This gives the possibility to use analytic solutions of Eq. (5) of domains which coincide with $G$ only in the neighborhood of $\Delta r$. The example of Fig. 2 shows the left part of the domain from Fig. 1. Choosing $\Delta r$ in the shown way, the analytically known solution of an infinite sector (see appendix) can be used, as long as $t<\Delta r^{2} / \gamma^{2} D$.

Hence, if the particle is neither annihilated nor absorbed within $\Delta t$, the particle will stay diffusive and a random pair $(\mathbf{r}, \Delta t)$ must be created for the particle's new position. In order to avoid repetitions, we skip the pseudo-code details here, as they will be shown in the next section in a more general case.

In most situations it is much better to use the subset method as its time-increments are generally much larger. But in a situation like the one sketched in Fig. 2 the particle is very close to the reflecting boundaries and no suitable large domain $G^{\prime}$ is available. In consequence, $G^{\prime}$ would be very small, leading to a very small $t_{b}$ on average.

\section{INHOMOGENEOUS ANNIHILATION RATE}

The last section showed how to find a solution for an arbitrary domain $G$ by solving the problem in several steps in smaller domains $G^{\prime}$. Hence, without loss of generality, we now assume the ability to sample random numbers according to $\rho_{b}^{D}, \rho_{f}^{D}$ and $\rho_{n}^{D}$ directly.

If the annihilation rate becomes inhomogeneous, Eq. (91) is not a solution of (1) anymore. The annihilationtime is now correlated to the complete path of the particle, thus the method presented in the previous section will not work. In this section we present a way to overcome this problem for arbitrary rates $k(\mathbf{r}, t)$ without any additional approximations. The following method starts with the pair $\left(\mathbf{r}_{0}, t_{0}\right)$ and generates a series of random pairs $\left(\mathbf{r}_{i}, t_{i}\right)$. The last pair of this series will become the new $(\mathbf{r}, t)$.

For all $t>t_{0}$ we define the spatially homogeneous but time dependent upper bound for the annihilation rates

$$
k_{m}(t)=\max _{\mathbf{r} \in G}\{k(\mathbf{r}, t)\}
$$

The density $\rho_{a}^{D}$ with the rate $k_{m}(t)$ is denoted by $\rho_{m}$ in the following:

$$
\rho_{m}\left(t \mid t_{0}\right)=-\frac{d}{d t}\left[e^{-\int_{t_{0}}^{t} k_{m}\left(t^{\prime}\right) d t^{\prime}}\right]
$$

We sample a candidate pair $\left(\mathbf{r}_{1}, t_{1}\right)$ as shown in Algorithm [1 For $\mathbf{r}_{1} \in \partial G$ the particle is absorbed at the boundary, i.e. the first candidate is accepted. Otherwise we compare the ratio $k\left(\mathbf{r}_{1}, t_{1}\right) / k_{m}\left(t_{1}\right)$ to a uniformly distributed random number $x$ in $[0,1]$. If $k\left(\mathbf{r}_{1}, t_{1}\right) / k_{m}\left(t_{1}\right) \geq$ $x$, the particle is annihilated, i.e. the first candidate is also accepted, else we generate a new candidate pair $\left(\mathbf{r}_{2}, t_{2}\right)$ under the condition of having been at position $\mathbf{r}_{1}$ at time $t_{1}$. This can be continued until the particle is absorbed at the boundary of $G$ or annihilated.

The algorithm can also be used to sample the complete probability density $P\left(\mathbf{r}, t \mid \mathbf{r}_{0}, t_{0}\right)$ : If no candidate is accepted until an arbitrarily chosen time $t_{\max }=t$ is reached, the algorithm returns a random position of the still diffusive particle, i.e a pair $\left(\mathbf{r}, t_{\max }\right)$ whose statistics is given by $P\left(\mathbf{r}, t_{\max } \mid \mathbf{r}_{0}, t_{0}\right)$. Also in case one wants to use the maximum distance method, the time $t_{\max }$ has to be chosen appropriately. If a break at $t_{\max }$ is not wanted, one simply sets $t_{\max }=\infty$. A pseudo-code description is shown in Algorithm 3

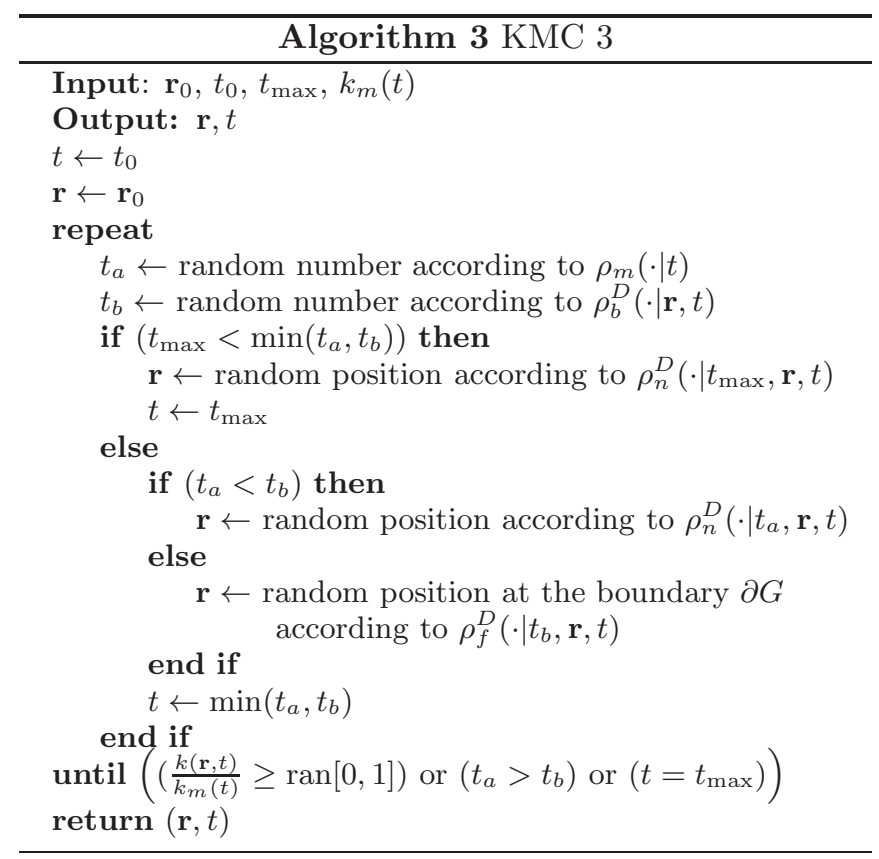




\section{A. Proof of correctness of Algorithm 3}

The basic mechanism by which the algorithm handles a spatially varying annihilation rate $k(\mathbf{r})$ is to generate trial annihilation positions using the propagator for a spatially constant (but maximal) annihilation rate $k_{m}$. The annihilation is then accepted with the local probability $k(\mathbf{r}) / k_{m}$ for. At first sight it appears counter-intuitive that this local procedure actually gives the correct statistics, since the probability to propagate a particle from $\mathbf{r}_{\mathbf{0}}$ to $\mathbf{r}$ depends on the complete annihilation rate landscape in between and around. Why is it sufficient to probe $k(\mathbf{r})$ locally at one or a few positions generated by the algorithm?

Before we answer this question rigorously by proving that it is indeed sufficient, we give an intuitive argument why one might expect the procedure to be correct: The stronger the spatial variation of $k(\mathbf{r})$ is in $G$ the larger the maximum rate $k_{m}$ will be. A large constant annihilation rate $k_{m}$ gives rise to a particle propagator that forbids large hops, which implies that the algorithm will produce many small intermediate hops and after each hop evaluates $k(\mathbf{r})$. In this way the algorithm explores stochastically the annihilation landscape on finer or coarser length scales depending on the variation of $k(\mathbf{r})$. If for instance $k(\mathbf{r})=0$ everywhere in $G$ except in a small restricted region, where it is $k(\mathbf{r})=k_{1}>0$, thus $k_{m}=k_{1}$. Then the algorithm will explore the complete region $G$ with a hop size that is characteristic for the restricted region with the non-vanishing annihilation rate. In the end this yields the correct statistics for the whole region, which we will prove now.

We will prove that the statistic of the output pairs $(\mathbf{r}, t)$ satisfy the probability flows $f_{a}$ and $f_{b}$ for $t<t_{\max }$. Then the case $t=t_{\max }$ (particle is still diffusive at time $\left.t_{\max }\right)$ occurs with the correct probability, too. We also prove that the statistic of output pairs $\left(\mathbf{r}, t_{\max }\right)$ coincides with $P\left(\mathbf{r}, t_{\max } \mid \mathbf{r}_{0}, t_{0}\right)$.

The algorithm will stop after a (unknown) number $i+1$ $(i \in \mathbb{N})$ of loop-runs (see Algorithm 3). The probability density for being annihilated after $i+1$ loop-runs at time $t$ at the position $\mathbf{r}$ is denoted by $A_{i}\left(\mathbf{r}, t \mid \mathbf{r}_{0}, t_{0}\right)$. Analogously the probability density for being absorbed at the boundary after $i+1$ loop-runs at time $t$ at the position $\mathbf{r}$ is denoted by $B_{i}\left(\mathbf{r}, t \mid \mathbf{r}_{0}, t_{0}\right)$. The probability density for stopping after $i+1$ loop-runs, still being in the diffusive state at $t_{\max }$ and being located at $\mathbf{r}$ is denoted by $W_{i}\left(\mathbf{r}, t_{\max } \mid \mathbf{r}_{0}, t_{0}\right)$. As the number of loop-runs is a disjoint decomposition, we can sum over $i$ to obtain the total densities for the corresponding events:

$$
\begin{aligned}
A\left(\mathbf{r}, t \mid \mathbf{r}_{0}, t_{0}\right) & =\sum_{i=0}^{\infty} A_{i}\left(\mathbf{r}, t \mid \mathbf{r}_{0}, t_{0}\right), \\
B\left(\mathbf{r}, t \mid \mathbf{r}_{0}, t_{0}\right) & =\sum_{i=0}^{\infty} B_{i}\left(\mathbf{r}, t \mid \mathbf{r}_{0}, t_{0}\right), \\
W\left(\mathbf{r}, t_{\max } \mid \mathbf{r}_{0}, t_{0}\right) & =\sum_{i=0}^{\infty} W_{i}\left(\mathbf{r}, t_{\max } \mid \mathbf{r}_{0}, t_{0}\right) .
\end{aligned}
$$

Starting with $i=0$, we compute $W_{i}, A_{i}, B_{i}$ :

$i=0$ :

$A_{0}$ : For this event $t_{a}$ has to be smaller than $t_{b}$, which delivers the second factor in the following product. The third factor belongs to the choice of the position and the last one arises from the exit-condition of the algorithm's loop:

$$
\begin{array}{r}
A_{0}\left(\mathbf{r}, t \mid \mathbf{r}_{0}, t_{0}\right)=\rho_{m}\left(t \mid t_{0}\right)\left(\int_{t}^{\infty} d t_{b} \rho_{b}^{D}\left(t_{b} \mid \mathbf{r}_{0}, t_{0}\right)\right) \\
\cdot \frac{P_{D}\left(\mathbf{r}, t \mid \mathbf{r}_{0}, t_{0}\right)}{\int_{G} d \mathbf{r}^{\prime} P_{D}\left(\mathbf{r}^{\prime}, t \mid \mathbf{r}_{0}, t_{0}\right)} \cdot \frac{k(\mathbf{r}, t)}{k_{m}(t)} \\
=k(\mathbf{r}, t) e^{-\int_{t_{0}}^{t} k_{m}\left(t^{\prime}\right) d t^{\prime}} P_{D}\left(\mathbf{r}, t \mid \mathbf{r}_{0}, t_{0}\right) .
\end{array}
$$

$B_{0}$ : An analogous procedure delivers

$$
\begin{aligned}
& B_{0}\left(\mathbf{r}, t \mid \mathbf{r}_{0}, t_{0}\right)=\rho_{b}^{D}\left(t \mid \mathbf{r}_{0}, t_{0}\right) e^{-\int_{t_{0}}^{t} k_{m}\left(t^{\prime}\right) d t^{\prime}} \\
& \frac{\nabla\left(P_{D}\left(\mathbf{r}, t \mid \mathbf{r}_{0}, t_{0}\right)\right) \cdot \mathbf{n}_{\mathbf{r}}}{\int_{\partial G} d F \nabla\left(P_{D}\left(\mathbf{r}, t \mid \mathbf{r}_{0}, t_{0}\right)\right) \cdot \mathbf{n}_{\mathbf{r}}} \\
& =-D e^{-\int_{t_{0}}^{t} k_{m}\left(t^{\prime}\right) d t^{\prime}} \nabla P_{D}\left(\mathbf{r}, t \mid \mathbf{r}_{0}, t_{0}\right) \cdot \mathbf{n}_{\mathbf{r}} \text {. }
\end{aligned}
$$

$W_{0}$ : If the particle reaches the time $t_{\max }$ in the first loop-run, $t_{a}$ and $t_{b}$ have to be larger than $t_{\max }$. Thus $W_{0}$ is the product of these two independent probabilities with the spatial density $\rho_{n}^{D}\left(\mathbf{r}, t_{\max } \mid \mathbf{r}_{0}, t_{0}\right)$ :

$$
\begin{gathered}
W_{0}\left(\mathbf{r}, t_{\max } \mid \mathbf{r}_{0}, t_{0}\right)=\left(\int_{t_{\max }}^{\infty} d t_{a} \rho_{m}\left(t_{a} \mid t_{0}\right)\right) \\
\cdot\left(\int_{t_{\max }}^{\infty} d t_{b} \rho_{b}^{D}\left(t_{b} \mid \mathbf{r}_{0}, t_{0}\right)\right) \cdot \frac{P_{D}\left(\mathbf{r}, t_{\max } \mid \mathbf{r}_{0}, t_{0}\right)}{\int_{G} d \mathbf{r}^{\prime} P_{D}\left(\mathbf{r}^{\prime}, t_{\max } \mid \mathbf{r}_{0}, t_{0}\right)} \\
=e^{-\int_{t_{0}}^{t_{\max }} k_{m}\left(t^{\prime}\right) d t^{\prime}} P_{D}\left(\mathbf{r}, t_{\max } \mid \mathbf{r}_{0}, t_{0}\right) .
\end{gathered}
$$

$i=1$ :

As the algorithm will pass the loop twice here, we have to sum/integrate over all weighted pairs $\left(\mathbf{r}_{\mathbf{1}}, t_{1}\right)$, which will be achieved in the first loop-run. Since the algorithm will only continue with a new loop if $t_{a}$ is smaller than $t_{b}$, for the first loop the factors and integrals look the same for all cases. The factors of the final loop can be taken from the individual factors of $i=0$ with the starting position $\mathbf{r}_{1}$ and the time $t_{1}$ instead of $\mathbf{r}_{0}$ and $t_{0}$. Defining the probability that the algorithm denies a candidate pair $(\mathbf{r}, t)$

$$
q(\mathbf{r}, t)=\left(1-\frac{k(\mathbf{r}, t)}{k_{m}(t)}\right),
$$

we get: 


$$
\begin{aligned}
A_{1}\left(\mathbf{r}, t \mid \mathbf{r}_{0}, t_{0}\right) & =\int_{t_{0}}^{t} d t_{a 1} \rho_{m}\left(t_{a 1} \mid t_{0}\right) \int_{t_{a 1}}^{\infty} d t_{b 1} \rho_{b}^{D}\left(t_{b 1} \mid \mathbf{r}_{0}, t_{0}\right) \int_{G} d \mathbf{r}_{1} \frac{P_{D}\left(\mathbf{r}_{1}, t_{a 1} \mid \mathbf{r}_{0}, t_{0}\right)}{\int_{G} d \mathbf{r}^{\prime} P_{D}\left(\mathbf{r}^{\prime}, t_{a 1} \mid \mathbf{r}_{0}, t_{0}\right)} q\left(\mathbf{r}_{1}, t_{a 1}\right) A_{0}\left(\mathbf{r}, t \mid \mathbf{r}_{1}, t_{a 1}\right) \\
& =k(\mathbf{r}, t) e^{-\int_{t_{0}}^{t} k_{m}\left(t^{\prime}\right) d t^{\prime}} \int_{t_{0}}^{t} d t_{a 1} \int_{G} d \mathbf{r}_{1} P_{D}\left(\mathbf{r}_{1}, t_{a 1} \mid \mathbf{r}_{0}, t_{0}\right) k_{m}\left(t_{a 1}\right) q\left(\mathbf{r}_{1}, t_{a 1}\right) P_{D}\left(\mathbf{r}, t \mid \mathbf{r}_{1}, t_{a 1}\right) \\
& =-D e^{-\int_{t_{0}}^{t} k_{m}\left(t^{\prime}\right) d t^{\prime}} \int_{t_{0}}^{t} d t_{a 1} \int_{G} d \mathbf{r}_{1} P_{D}\left(\mathbf{r}_{1}, t_{a 1} \mid \mathbf{r}_{0}, t_{0}\right) k_{m}\left(t_{a 1}\right) q\left(\mathbf{r}_{1}, t_{a 1}\right) \nabla P_{D}\left(\mathbf{r}, t \mid \mathbf{r}_{1}, t_{a 1}\right) \cdot \mathbf{n}_{\mathbf{r}} \\
B_{1}\left(\mathbf{r}, t \mid \mathbf{r}_{0}, t_{0}\right) & =\int_{t_{0}}^{t} d t_{a 1} \rho_{m}\left(t_{a 1} \mid t_{0}\right) \int_{t_{a 1}}^{\infty} d t_{b 1} \rho_{b}^{D}\left(t_{b 1} \mid \mathbf{r}_{0}, t_{0}\right) \int_{G} d \mathbf{r}_{1} \frac{P_{D}\left(\mathbf{r}_{1}, t_{a 1} \mid \mathbf{r}_{0}, t_{0}\right)}{\int_{G} d \mathbf{r}^{\prime} P_{D}\left(\mathbf{r}^{\prime}, t_{a 1} \mid \mathbf{r}_{0}, t_{0}\right)} q\left(\mathbf{r}_{1}, t_{a 1}\right) B_{0}\left(\mathbf{r}, t \mid \mathbf{r}_{1}, t_{a 1}\right) \\
W_{1}\left(\mathbf{r}, t_{\max } \mid \mathbf{r}_{0}, t_{0}\right) & (27) \\
=\int_{t_{0}}^{t_{\max }} d t_{a 1} \rho_{m}\left(t_{a 1} \mid t_{0}\right) & \int_{t_{a 1}}^{\infty} d t_{b 1} \rho_{b}^{D}\left(t_{b 1} \mid \mathbf{r}_{0}, t_{0}\right) \int_{G} d \mathbf{r}_{1} \frac{P_{D}\left(\mathbf{r}_{1}, t_{a 1} \mid \mathbf{r}_{0}, t_{0}\right)}{\int_{G} d \mathbf{r}^{\prime} P_{D}\left(\mathbf{r}^{\prime}, t_{a 1} \mid \mathbf{r}_{0}, t_{0}\right)} q\left(\mathbf{r}_{1}, t_{a 1}\right) W_{0}\left(\mathbf{r}, t_{\max } \mid \mathbf{r}_{1}, t_{a 1}\right) \\
& =e^{-\int_{t_{0}}^{t_{\max }} k_{m}\left(t^{\prime}\right) d t^{\prime}} \int_{t_{0}}^{t_{\max }} d t_{a 1} \int_{G} d \mathbf{r}_{1} P_{D}\left(\mathbf{r}_{1}, t_{a 1} \mid \mathbf{r}_{0}, t_{0}\right) k_{m}\left(t_{a 1}\right) q\left(\mathbf{r}_{1}, t_{a 1}\right) P_{D}\left(\mathbf{r}, t_{\max } \mid \mathbf{r}_{1}, t_{a 1}\right)
\end{aligned}
$$

For $i \geq 1$ we introduce the definitions

$$
\begin{array}{r}
h_{i}\left(\mathbf{r}, t \mid \mathbf{r}_{0}, t_{0}\right)=\int\left(\prod_{j=1}^{i} d t_{j}\right) k_{m}\left(t_{j}\right) \int_{G^{n}}\left(\prod_{k=1}^{i} d \mathbf{r}_{k}\right) Q_{i}\left(\left\{\left(\mathbf{r}_{l}, t_{l}\right)\right\}_{l=0 . . i}\right) P_{D}\left(\mathbf{r}, t \mid \mathbf{r}_{i}, t_{i}\right), \\
\text { with } \quad Q_{i}\left(\left\{\left(\mathbf{r}_{l}, t_{l}\right)\right\}_{l=0 . . i}\right)=\prod_{l=1}^{i} P_{D}\left(\mathbf{r}_{l}, t_{l} \mid \mathbf{r}_{l-1}, t_{l-1}\right) \cdot q\left(\mathbf{r}_{l}, t_{l}\right) .
\end{array}
$$

Finally, defining

$$
h_{0}\left(\mathbf{r}, t \mid \mathbf{r}_{0}, t_{0}\right)=P_{D}\left(\mathbf{r}, t \mid \mathbf{r}_{0}, t_{0}\right)
$$

one inductively gets for $i \geq 0$ :

$$
\begin{aligned}
A_{i}\left(\mathbf{r}, t \mid \mathbf{r}_{0}, t_{0}\right) & =k(\mathbf{r}, t) e^{-\int_{t_{0}}^{t} k_{m}\left(t^{\prime}\right) d t^{\prime}} h_{i}\left(\mathbf{r}, t \mid \mathbf{r}_{0}, t_{0}\right) \\
B_{i}\left(\mathbf{r}, t \mid \mathbf{r}_{0}, t_{0}\right) & =-D e^{-\int_{t_{0}}^{t} k_{m}\left(t^{\prime}\right) d t^{\prime}} \nabla h_{i}\left(\mathbf{r}, t \mid \mathbf{r}_{0}, t_{0}\right) \cdot \mathbf{n}_{\mathbf{r}} \\
W_{i}\left(\mathbf{r}, t_{\max } \mid \mathbf{r}_{0}, t_{0}\right) & =e^{-\int_{t_{0}}^{t_{\max }} k_{m}\left(t^{\prime}\right) d t^{\prime}} h_{i}\left(\mathbf{r}, t_{\max } \mid \mathbf{r}_{0}, t_{0}\right)
\end{aligned}
$$

Hence, the total probability densities can be written as

$$
\begin{aligned}
A\left(\mathbf{r}, t \mid \mathbf{r}_{0}, t_{0}\right) & =k(\mathbf{r}, t) \tilde{P}\left(\mathbf{r}, t \mid \mathbf{r}_{0}, t_{0}\right) \\
B\left(\mathbf{r}, t \mid \mathbf{r}_{0}, t_{0}\right) & =-D \nabla \tilde{P}\left(\mathbf{r}, t \mid \mathbf{r}_{0}, t_{0}\right) \cdot \mathbf{n}_{\mathbf{r}}, \\
W\left(\mathbf{r}, t_{\max } \mid \mathbf{r}_{0}, t_{0}\right) & =\tilde{P}\left(\mathbf{r}, t_{\max } \mid \mathbf{r}_{0}, t_{0}\right)
\end{aligned}
$$

with

$$
\tilde{P}\left(\mathbf{r}, t \mid \mathbf{r}_{0}, t_{0}\right)=e^{-\int_{t_{0}}^{t} k_{m}\left(t^{\prime}\right) d t^{\prime}} \sum_{i=0}^{\infty} h_{i}\left(\mathbf{r}, t \mid \mathbf{r}_{0}, t_{0}\right)
$$

Comparing this with the definitions of $f_{a}$ and $f_{b}$, it remains to show that $\tilde{P}\left(\mathbf{r}, t \mid \mathbf{r}_{0}, t_{0}\right) \stackrel{!}{=} P\left(\mathbf{r}, t \mid \mathbf{r}_{0}, t_{0}\right)$, i.e. $\tilde{P}\left(\mathbf{r}, t \mid \mathbf{r}_{0}, t_{0}\right)$ has to satisfy Eq. (11) with the initial condition $\tilde{P}\left(\mathbf{r}, t_{0} \mid \mathbf{r}_{0}, t_{0}\right)=\delta\left(\mathbf{r}-\mathbf{r}_{0}\right)$. As all $h_{i}$ with $i \geq 1$ vanish for $t=t_{0}$, the initial condition is simply fulfilled by the definition of $h_{0}$. For the time-derivative of $h_{i}$ one inductively gets for $i \geq 1$ : 


$$
\begin{aligned}
\dot{h}_{i}\left(\mathbf{r}, t \mid \mathbf{r}_{0}, t_{0}\right)= & k_{m}(t) q(\mathbf{r}, t) \cdot h_{i-1}\left(\mathbf{r}, t \mid \mathbf{r}_{0}, t_{0}\right) \\
& +\int\left(\prod_{j=1}^{i} d t_{j}\right) k_{m}\left(t_{j}\right) \int_{G^{n}}\left(\prod_{k=1}^{i} d \mathbf{r}_{k}\right) Q_{i}\left(\left\{\left(\mathbf{r}_{l}, t_{l}\right)\right\}_{l=0 . . i}\right) \dot{P}_{D}\left(\mathbf{r}, t \mid \mathbf{r}_{i}, t_{i}\right) \\
t_{0} \leq t_{1} \leq \ldots \leq t_{i} \leq t &
\end{aligned}
$$

Hence, the time-derivative of $\tilde{P}\left(\mathbf{r}, t \mid \mathbf{r}_{0}, t_{0}\right)$ satisfies

$$
\begin{aligned}
\frac{\partial \tilde{P}\left(\mathbf{r}, t \mid \mathbf{r}_{0}, t_{0}\right)}{\partial t}= & -k_{m}(t) \tilde{P}\left(\mathbf{r}, t \mid \mathbf{r}_{0}, t_{0}\right)+e^{-\int_{t_{0}}^{t} k_{m}\left(t^{\prime}\right) d t^{\prime}}\left\{\dot{P}_{D}\left(\mathbf{r}, t \mid \mathbf{r}_{0}, t_{0}\right)+\sum_{i=1}^{\infty} \dot{h}_{i}\left(\mathbf{r}, t \mid \mathbf{r}_{0}, t_{0}\right)\right\} \\
= & -k_{m}(t) \tilde{P}\left(\mathbf{r}, t \mid \mathbf{r}_{0}, t_{0}\right)+e^{-\int_{t_{0}}^{t} k_{m}\left(t^{\prime}\right) d t^{\prime}}\left\{\dot{P}_{D}\left(\mathbf{r}, t \mid \mathbf{r}_{0}, t_{0}\right)+k_{m}(t) q(\mathbf{r}, t) \sum_{i=1}^{\infty} h_{i-1}\left(\mathbf{r}, t \mid \mathbf{r}_{0}, t_{0}\right)\right. \\
& \left.+\sum_{i=1}^{\infty} \int\left(\prod_{j=1}^{i} d t_{j}\right) k_{m}\left(t_{j}\right) \int_{G^{n}}\left(\prod_{k=1}^{i} d \mathbf{r}_{k}\right) Q_{i}\left(\left\{\left(\mathbf{r}_{l}, t_{l}\right)\right\}_{l=0 . . i}\right) \dot{P}_{D}\left(\mathbf{r}, t \mid \mathbf{r}_{i}, t_{i}\right)\right\} \\
= & -k(r, t) \tilde{P}\left(\mathbf{r}, t \mid \mathbf{r}_{0}, t_{0}\right)+e^{-\int_{t_{0}}^{t} k_{m}\left(t^{\prime}\right) d t^{\prime}}\left\{\dot{P}_{D}\left(\mathbf{r}, t \mid \mathbf{r}_{0}, t_{0}\right)+\right. \\
& \left.\sum_{i=1}^{\infty} \int\left(\prod_{j=1}^{i} d t_{j}\right) k_{m}\left(t_{j}\right) \int_{G^{n}}\left(\prod_{k=1}^{i} d \mathbf{r}_{k}\right) Q_{i}\left(\left\{\left(\mathbf{r}_{l}, t_{l}\right)\right\}_{l=0 . . i}\right) \dot{P}_{D}\left(\mathbf{r}, t \mid \mathbf{r}_{i}, t_{i}\right)\right\}
\end{aligned}
$$

Thus, using Eq. (5), it follows:

$$
\begin{aligned}
\frac{\partial \tilde{P}\left(\mathbf{r}, t \mid \mathbf{r}_{0}, t_{0}\right)}{\partial t} & =-k(\mathbf{r}, t) \tilde{P}\left(\mathbf{r}, t \mid \mathbf{r}_{0}, t_{0}\right)+e^{-\int_{t_{0}}^{t_{\max }} k_{m}\left(t^{\prime}\right) d t^{\prime}} D \Delta\left\{\sum_{i=0}^{\infty} h_{i}\left(\mathbf{r}, t \mid \mathbf{r}_{0}, t_{0}\right)\right\} \\
& =-k(\mathbf{r}, t) \tilde{P}\left(\mathbf{r}, t \mid \mathbf{r}_{0}, t_{0}\right)+D \Delta \tilde{P}\left(\mathbf{r}, t \mid \mathbf{r}_{0}, t_{0}\right)
\end{aligned}
$$

which is exactly Eq. (11). Hence, the correctness of the Algorithm 3 is proven.

\section{EXAMPLE}

This section presents a two-dimensional application example of the algorithm. It is designed to demonstrate how the algorithm handles a situation in which its correctness is most counter-intuitive: We choose the annihilation rate to be non-vanishing just inside a restricted region, a circle, where it oscillates in time and varies spatially. For a chosen test-setup, we compare its results with the solution of a commercial FEM (finite element method) routine.

At $t_{0}=0$ the diffusing particle $(D=1)$ is located at the position $\mathbf{r}_{0}=(0 ; 5)$ within a rectangle of size $10 \times 5$. The right boundary is chosen to be absorbing, all other boundaries are reflecting. A strongly anisotropic time dependent annihilation rate $k(\mathbf{r}, t)$ is chosen to be

$$
k(\mathbf{r}, t)=\left\{\begin{array}{cc}
3\left|\cos ^{3}\left(\frac{t}{2}\right)\right| \cdot\left(c^{2}-\|\mathbf{r}-\mathbf{z}\|^{2}\right), & \|\mathbf{r}-\mathbf{z}\|<c \\
0, & \|\mathbf{r}-\mathbf{z}\| \geq c
\end{array},\right.
$$

with $\mathbf{z}=(5 ; 1.25)$ and $c=1.25$. Fig. 3 presents a sketch of the described setup.

On the one hand the problem has been solved numerically by applying a commercial FEM solver with a very fine triangulation (> 600000 elements) to Eq. (1). In the following this solution is denoted by $P_{F}(\mathbf{r}, t)$. On the other hand the Monte-Carlo algorithm has been applied to the problem in $4.2 \times 10^{8}$ samples. In principle it is not necessary to use the subset method here, as the analytic solution of Eq. (5) is known for the rectangle (see appendix), from which all occurring densities can be sampled. Nevertheless it has been used, as it increases the speed of the algorithm dramatically: For all highly anisotropic annihilation rates the ratio $k / k_{m}$ in Algo- 


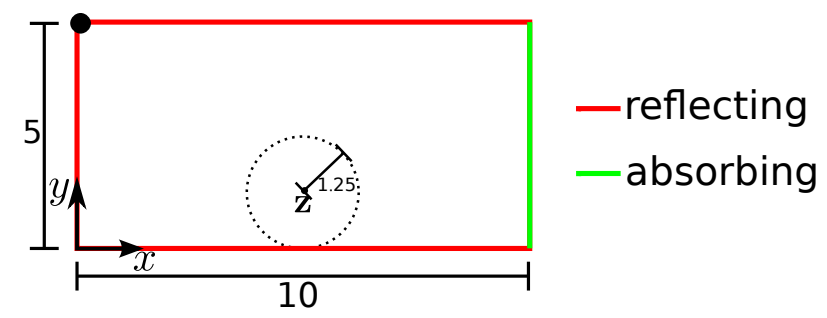

FIG. 3: sketch of the simulation setup: The particle starts its diffusive motion at the upper left corner. It can either be absorbed at the right wall or annihilated within the drawn circle.

rithm 3 will mostly be very small (in our case even 0 ). Hence, a lot of loop runs with just small time incrementations will on average be needed for an event. Restricting the movement of the particle temporally to a subset of $G$ (subset method) ensures the possibility of choosing a smaller $k_{m}(t)$, which reduces the number of loop-runs in Algorithm 3 dramatically. Using this in our case it takes around 40 minutes for $10^{6}$ samples on a single core with $3.4 \mathrm{GHz}$.

Firstly, the relative frequencies for the times of an event and the kind of the event were counted. $S_{M C}(t)$ denotes the relative frequency of having had no event until time $t$. It has to be compared with the value of $S_{F}(t)=1-\int_{0}^{t} \rho_{e}\left(t^{\prime} \mid \mathbf{r}_{0}, t_{0}\right) d t^{\prime}$, which was derived numerically from the FEM solution. $A_{M C}(t)$ denotes the relative frequency of having had an annihilation event before time $t$. It is compared to $A_{F}(t)=\int_{0}^{t} \alpha\left(t^{\prime} \mid \mathbf{r}_{0}, t_{0}\right) d t^{\prime}$. $B_{M C}(t)$ denotes the relative frequency of being absorbed at the right boundary before time $t$. It is compared to $B_{F}(t)=\int_{0}^{t} \beta\left(t^{\prime} \mid \mathbf{r}_{0}, t_{0}\right) d t^{\prime}$. A plot of these quantities is shown in Fig. 4]

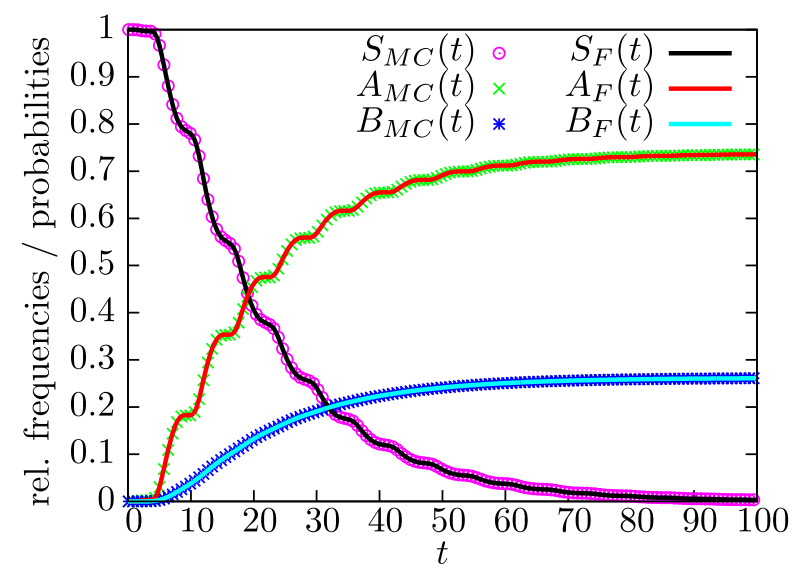

FIG. 4: Comparison of the relative frequencies derived from Algorithm 3 and the numerically integrated probabilities for the events of still being diffusive $(S)$, having already been annihilated $(A)$ and having already been absorbed at the right wall $(B)$.
It shows an almost perfect coincidence of all corresponding quantities. The maximum relative deviation is about $1 \%$ in all curves. Keeping in mind that $S_{F}(t)$, $A_{F}(t)$ and $B_{F}(t)$ are calculated by a numerical timeintegration of a numerical spatial integration of a numerical solution of Eq. (11), these small deviations are explainable. More precisely, $S_{F}(t)+A_{F}(t)+B_{F}(t)=1$ has to hold for all times, but the numerical discrepancy in this sum is also about $1 \%$ at maximum.

Secondly, we want to compare the spatial distribution of the particle's position from the KMC algorithm to $P_{F}(\mathbf{r}, t)$ for three characteristic times: $t_{1}=5, t_{2}=10$, $t_{3}=20$. Hence, the rectangle is divided in $2 N \times N$ squares $s_{x y}\left(x=i \cdot \frac{5}{N}, y=j \cdot \frac{5}{N}, i=0,1, \ldots 2 N-1, j=\right.$ $0,1, \ldots N-1)$ and the relative frequency $h_{x y}(t)$ for being at the square $s_{x y}$ is counted for $t_{1}, t_{2}$ and $t_{3}$. Technically this has been done by setting $t_{\max }=t_{i}(i \in\{1,2,3\})$ in Algorithm 3. The quotient of $h_{x y}(t)$ and the area of a square element is denoted by $P_{x y}(t)$. This density converges to the solution of Eq. (11) in the limits of increasing sample numbers and $N \rightarrow \infty$. The upper panel in Fig. 5 shows the density $P_{x y}(t)$ for the chosen times in a $3 \mathrm{~d}$ plot for $N=50$. In order to illustrate the influence of the annihilation within the circle, the projection on the bottom shows isolines by discretising the density into intervals.

- $t_{1}=5$ : The probability density of the particle is still centered around the starting position in the upper left corner. Nevertheless its shape is already influenced by the annihilation within the circle.

- $t_{2}=10$ : At time $t_{2}$ there has been almost no annihilation for a short period (slope of the red line in Fig. 44). Hence, diffusion almost equilibrated the density gradient in $\mathrm{y}$-direction, generated by the annihilation within the time-interval $[5 ; 8]$.

- $t_{3}=20$ : At time $t_{3}$ relatively strong annihilation takes place, which even leads to a local minimum of $P_{x y}$ within the circle.

In order to quantify the local differences between the KMC result and the FEM result, we choose squares of size $0.2 \times 0.2(N=25)$. A measure for the spatially resolved relative deviation is

$$
\Delta_{x y}(t)=\frac{h_{x y}(t)-\int_{x}^{x+0.2} d x^{\prime} \int_{y}^{y+0.2} d y^{\prime} P_{F}\left(\mathbf{r}^{\prime}, t\right)}{\int_{x}^{x+0.2} d x^{\prime} \int_{y}^{y+0.2} d y^{\prime} P_{F}\left(\mathbf{r}^{\prime}, t\right)} .
$$

The lower panel of Fig. 5 shows $\Delta_{x y}(t)$. For all times the deviations are small and in the range of the numerical expectation: For $4.2 \times 10^{8}$ samples and $(2 N)^{2}=10^{4}$ plaquettes one expects ca. $10^{4}$ samples per plaquette and thus statistical fluctuation of the order of $10^{2}$, i.e. relative fluctuation in the 1 percent range, which is what the lower panel of Fig. 5 confirms.

On the right side of the simulation rectangle, where the absorbing boundary is located, the statistical error is larger for small times, since the density is still centered 

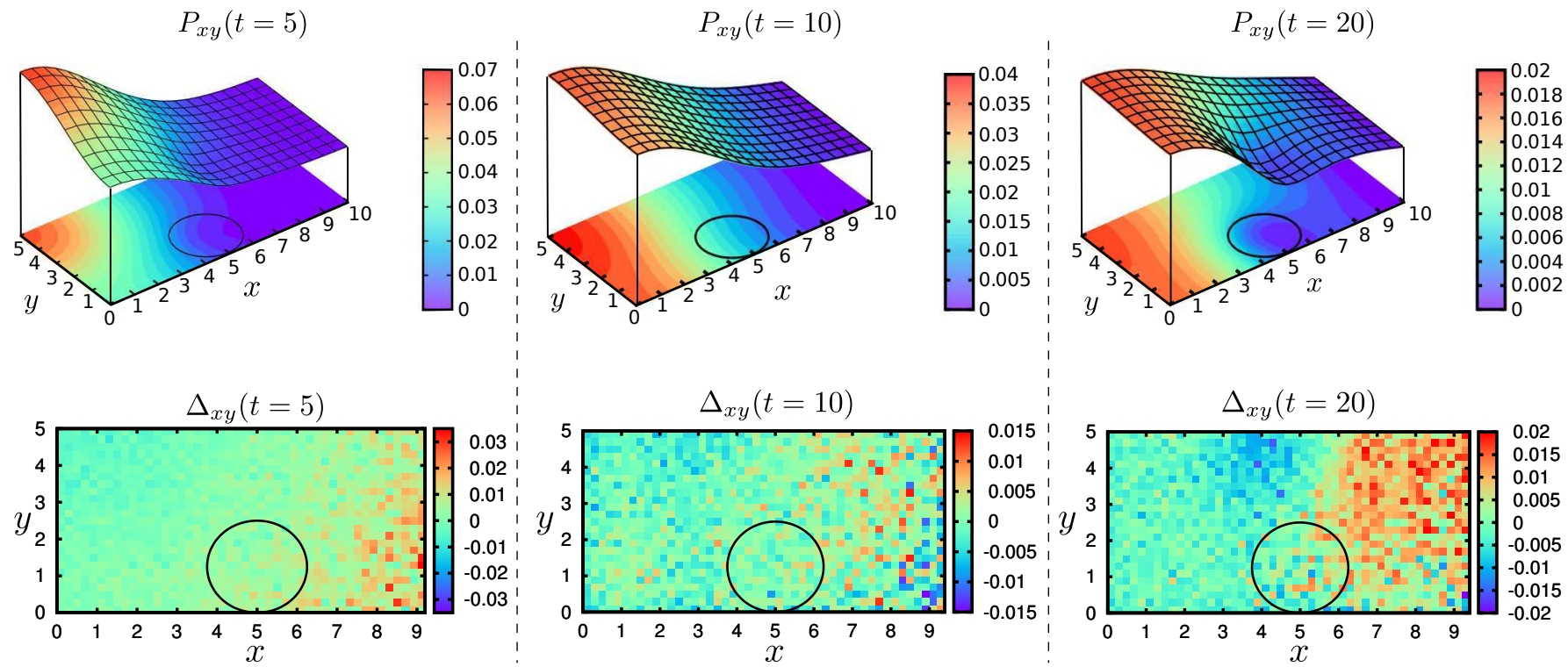

FIG. 5: Top: The probability density $P_{x y}(t)=P\left(\mathbf{r}, t \mid \mathbf{r}_{0}, t_{0}\right)$ for the two-dimensional geometry depicted in Fig. 3 and the annihilation rate given by Eq. (44) generated by the intermediate positions of Algorithm 3 for the times $t_{1}=5, t_{2}=10$ and $t_{3}=20$. The position of the annihilation zone with oscillating strength is indicated by the full circle. Bottom: Relative difference $\Delta_{x y}(t)$ between the Monte Carlo result $P_{x y}(t)$ and the numerical solution of the corresponding annihilation-diffusion Eq. (11) for the times $t_{1}=5, t_{2}=10$ and $t_{3}=20$.

around the starting point in the upper left corner, giving a region $(x>8)$ with very small values of $h_{x y}(t)$. But the aim of the Algorithm 3 is not the stochastic solution of Eq. (1), for which finite element methods are suitable. The aim of the algorithm is to sample correctly next events (annihilations or first-passages of boundaries) according to Eq. (11), which can not be handled by a FEM routine. The example demonstrates, that this is possible, even in cases of highly anisotropic and time dependent annihilation rates.

\section{DISCUSSION}

We have presented an algorithm that samples correctly the probability distribution of a diffusing particle with a space dependent annihilation or transformation rate $k(\mathbf{r})$ for arbitrary domains. Together with first-passage time methods it can serve as the basic building block for a kinetic Monte Carlo algorithm simulating a general many-particle reaction-diffusion system.

The basic idea is to generate trial moves with the exactly known single particle Green's function for a spatially constant annihilation rate $k_{m}$, which is the maximum of $k(\mathbf{r})$ in the current protecting domain. With probability $k(\mathbf{r}) / k_{m}$ the particle is annihilated at the trial position $\mathbf{r}$, otherwise a new trial move with initial position $\mathbf{r}$ is generated. The iteration proceeds until either the particle is annihilated or the boundary of the protecting domain is reached. In this paper we proved rigorously the correctness of this algorithm and demonstrated its numerical accuracy and efficiency with an illustrative example.

Important applications with a spatially varying transformation rate include continuum models for intracellular transport (or more generally intermittent search strategies [13]). In intracellular transport particles (proteins, organelles) can switch between free diffusion and ballistic motion by molecular motor assisted movement along cytoskeleton filaments. The density of filaments in the space direction $\Omega, \rho_{\Omega}(\mathbf{r}, t)$, is generally very inhomogeneous in space and sometimes even varies over time (for instance during cell polarization). This situation can be described by the Fokker-Planck equation for the probability densities $P_{0}(\mathbf{r}, t)$ and $P_{\Omega}(\mathbf{r}, t)$ for diffusing particles and particles that move with a constant velocity $\mathbf{v}_{\Omega}$ in direction $\Omega$, respectively [9]:

$$
\begin{aligned}
\frac{\partial}{\partial t} P_{0}(\mathbf{r}, t)= & D \Delta P_{0}(\mathbf{r}, t)-\gamma P_{0}(\mathbf{r}, t) \int d \Omega \rho_{\Omega}(\mathbf{r}, t) \\
& +\gamma^{\prime} \int d \Omega P_{\Omega}(\mathbf{r}, t) \\
\frac{\partial}{\partial t} P_{\Omega}(\mathbf{r}, t)= & -\nabla \cdot\left(\mathbf{v}_{\Omega} P_{\Omega}(\mathbf{r}, t)\right)+\gamma \rho_{\Omega}(\mathbf{r}, t) P_{0}(\mathbf{r}, t) \\
& -\gamma^{\prime} P_{\Omega}(\mathbf{r}, t),
\end{aligned}
$$

where $\gamma$ and $\gamma^{\prime}$ are the attachment and detachment rates (to and from filaments), respectively. The freely diffusing particle sees a total annihilation rate $k(\mathbf{r}, t)=\gamma \int d \Omega \rho_{\Omega}(\mathbf{r}, t)$, with which it is transformed into a ballistically moving particle with a randomly chosen direction $\Omega$ (and velocity $\mathbf{v}_{\Omega}$ ) with probability 
$\rho_{\Omega}(\mathbf{r}, t) / \int d \Omega \rho_{\Omega}(\mathbf{r}, t)$. The algorithm presented in this paper handles a Monte Carlo simulation of the diffusion process described by (45), whereas the implementation of the ballistic motion (46) is straightforward.

\section{Appendix A}

This appendix presents some analytic solutions of Eq. (5), which have mostly been taken from [12]. Furthermore, it derives expressions for sampling according to the densities $\rho_{b}^{D}, \rho_{f}^{D}, \rho_{n}^{D}$ in cases where this might not be obvious anymore. We list only frequently used domains in one, two and three dimensions.

\section{Particle on the interval $[0, L]$}

- absorbing on both sides:

$P_{D}\left(x, t \mid x_{0}, t_{0}\right)=\frac{2}{L} \sum_{n=1}^{\infty} e^{-k_{n}^{2} D\left(t-t_{0}\right)} \sin \left(k_{n} x\right) \sin \left(k_{n} x_{0}\right)$,

with $k_{n}=\frac{n \pi}{L}$.

Expressions for the probability densities $\rho_{b}^{D}, \rho_{f}^{D}, \rho_{n}^{D}$ and the corresponding distribution functions $F_{b}^{D}, F_{f}^{D}$, $F_{n}^{D}$ can be derived analytically.

- reflecting on the left and absorbing on the right side:

$P_{D}\left(x, t \mid x_{0}, t_{0}\right)=\frac{2}{L} \sum_{n=0}^{\infty} e^{-k_{n}^{2} D\left(t-t_{0}\right)} \cos \left(k_{n} x\right) \cos \left(k_{n} x_{0}\right)$,

with $k_{n}=\frac{(2 n+1) \pi}{2 L}$.

Expressions for the probability densities $\rho_{b}^{D}, \rho_{n}^{D}$ and the corresponding distribution functions $F_{b}^{D}, F_{n}^{D}$ are analytically derivable. As there is only $x=L$ for the particle to leave the domain, it follows $\rho_{f}^{D}\left(0 \mid t, x_{0}, t_{0}\right)=$ 0 and $\rho_{f}^{D}\left(L \mid t, x_{0}, t_{0}\right)=1$.

- reflecting on both sides:

$$
\begin{aligned}
& P_{D}\left(x, t \mid x_{0}, t_{0}\right)= \\
& \frac{1}{L}\left(1+2 \sum_{n=0}^{\infty} e^{-k_{n}^{2} D\left(t-t_{0}\right)} \cos \left(k_{n} x\right) \cos \left(k_{n} x_{0}\right)\right),
\end{aligned}
$$

with $k_{n}=\frac{n \pi}{L}$.

Expressions for the probability density $\rho_{n}^{D}$ and the corresponding distribution function $F_{n}^{D}$ can be derived analytically.

\section{Particle in a rectangle $[0, a] \times[0, b]$ and in a cuboid $[0, a] \times[0, b] \times[0, c]$}

If the boundary conditions do not vary along each side, $P_{D}$ factorizes:

$P_{D}=P_{D}^{a}\left(x, t \mid x_{0}, t_{0}\right) \cdot P_{D}^{b}\left(y, t \mid y_{0}, t_{0}\right) \quad\left(\cdot P_{D}^{c}\left(z, t \mid z_{0}, t_{0}\right)\right)$, where $P_{D}^{a}, P_{D}^{b}\left(P_{D}^{c}\right)$ are given by solutions for intervals from the subsection above. Depending on the boundary conditions, $\rho_{b}^{D}$ is sampled by generating a random time for every coordinate, where there is at least one absorbing boundary. The smallest of these times has to be returned as $t_{b}$. The particle reaches the boundary in the corresponding coordinate. All other quantities are sampled as above.

\section{Particle in a circle of radius $R$}

- absorbing boundary:

$$
\begin{gathered}
P_{D}\left(r, \varphi, t \mid r_{0}, \varphi_{0}, t_{0}\right)=\frac{1}{\pi R^{2}}\left[\sum_{n=-\infty}^{\infty} \cos \left(n\left(\varphi-\varphi_{0}\right)\right)\right. \\
\left.\cdot \sum_{\alpha_{n}} e^{-\alpha_{n}^{2} \frac{D\left(t-t_{0}\right)}{R^{2}}} \frac{J_{n}\left(\alpha_{n} \frac{r}{R}\right) J_{n}\left(\alpha_{n} \frac{r_{0}}{R}\right)}{J_{n}^{\prime}\left(\alpha_{n}\right)^{2}}\right],
\end{gathered}
$$

where $\sum_{\alpha_{n}}$ denotes the infinite sum over all positive roots $\alpha_{n}$ of the Bessel function $J_{n}\left(\alpha_{n}\right)=0$.

The density of finding the particle at an arbitrary angle at radius $r$ is then given by

$$
\begin{aligned}
\rho_{r}\left(r, t \mid r_{0}, t_{0}\right) & =\int_{0}^{2 \pi} P_{D}\left(r, \varphi, t \mid r_{0}, \varphi_{0}, t_{0}\right) r d \varphi \\
& =\frac{2}{R^{2}} \sum_{\alpha_{0}} e^{-\alpha_{0}^{2} \frac{D\left(t-t_{0}\right)}{R^{2}}} r \frac{J_{0}\left(\alpha_{0} \frac{r}{R}\right) J_{0}\left(\alpha_{0} \frac{r_{0}}{R}\right)}{J_{1}\left(\alpha_{0}\right)^{2}} .
\end{aligned}
$$

and the corresponding distribution function is given by

$$
\begin{aligned}
F_{r}\left(r, t \mid r_{0}, t_{0}\right) & =\int_{0}^{r} d r^{\prime} \rho_{r}\left(r^{\prime}, t \mid r_{0}, t_{0}\right) \\
& =\frac{2}{R} \sum_{\alpha_{0}} e^{-\alpha_{0}^{2} \frac{D\left(t-t_{0}\right)}{R^{2}}} r \frac{J_{1}\left(\alpha_{0} \frac{r}{R}\right) J_{0}\left(\alpha_{0} \frac{r_{0}}{R}\right)}{\alpha_{0} J_{1}\left(\alpha_{0}\right)^{2}} .
\end{aligned}
$$

Hence, the distribution function belonging to $\rho_{b}^{D}$ is given by

$$
F_{b}^{D}\left(t \mid r_{0}, t_{0}\right)=1-2 \sum_{\alpha_{0}} e^{-\alpha_{0}^{2} \frac{D\left(t-t_{0}\right)}{R^{2}}} \frac{J_{0}\left(\alpha_{0} \frac{r_{0}}{R}\right)}{\alpha_{0} J_{1}\left(\alpha_{0}\right)} .
$$

Analytic expressions for all quantities depending on $\varphi$ which are needed, are straightforwardly derivable by integrating the cos-functions.

Having precomputed the values of $\alpha_{n}$, random numbers are sampled by inverting the occurring distribution functions numerically.

For a particle starting in the center of the circle the $\varphi$ dependence becomes uniformly distributed in the interval $\left[0,2 \pi\left[\right.\right.$ and $F_{b}^{D}$ simplifies to

$$
F_{b}^{D}\left(t \mid 0, t_{0}\right)=1-2 \sum_{\alpha_{0}} e^{-\alpha_{0}^{2} \frac{D\left(t-t_{0}\right)}{R^{2}}} \frac{1}{\alpha_{0} J_{1}\left(\alpha_{0}\right)},
$$

which is used to derive Eq. (14). 
- reflecting boundary:

$$
\begin{gathered}
P_{D}\left(r, \varphi, t \mid r_{0}, \varphi_{0}, t_{0}\right)=\frac{1}{\pi R^{2}}\left[1+\sum_{n=-\infty}^{\infty} \cos \left(n\left(\varphi-\varphi_{0}\right)\right)\right. \\
\left.\cdot \sum_{\alpha_{n}} e^{-\alpha_{n}^{2} \frac{D\left(t-t_{0}\right)}{R^{2}}} \frac{J_{n}\left(\alpha_{n} \frac{r}{R}\right) J_{n}\left(\alpha_{n} \frac{r_{0}}{R}\right)}{\left(1-\frac{n^{2}}{\alpha_{n}^{2}}\right) J_{n}\left(\alpha_{n}\right)^{2}}\right],
\end{gathered}
$$

where $\sum_{\alpha_{n}}$ denotes the infinite sum over all positive roots $\alpha_{n}$ of $J_{n}^{\prime}\left(\alpha_{n}\right)=0$.

The density of finding the particle at an arbitrary angle at radius $r$ is then given by

$$
\begin{aligned}
& \rho_{r}\left(r, t \mid r_{0}, t_{0}\right)=\int_{0}^{2 \pi} \rho\left(r, \varphi, t \mid r_{0}, \varphi_{0}\right) r d \varphi \\
& =\frac{2}{R^{2}}\left[r+\sum_{\alpha_{0}} e^{-\alpha_{0}^{2} \frac{D\left(t-t_{0}\right)}{R^{2}}} r \frac{J_{0}\left(\alpha_{0} \frac{r}{R}\right) J_{0}\left(\alpha_{0} \frac{r_{0}}{R}\right)}{J_{0}\left(\alpha_{0}\right)^{2}}\right]
\end{aligned}
$$

and the corresponding distribution function is given by

$$
\begin{aligned}
& F_{r}\left(r, t \mid r_{0}, t_{0}\right)= \\
& \quad \frac{r^{2}}{R^{2}}+\frac{2}{R} \sum_{\alpha_{0}} e^{-\alpha_{0}^{2} \frac{D\left(t-t_{0}\right)}{R^{2}}} r \frac{J_{1}\left(\alpha_{0} \frac{r}{R}\right) J_{0}\left(\alpha_{0} \frac{r_{0}}{R}\right)}{\alpha_{0} J_{0}\left(\alpha_{0}\right)^{2}} .
\end{aligned}
$$

A distribution function for the angle $\varphi$ under the condition of being at radius $r$ can be derived straightforwardly by integrating the cos-functions. Having precomputed the values of $\alpha_{n}, r$ and $\varphi$ are sampled by a numerical inversion of the distribution functions. For a particle starting in the center of the circle the $\varphi$ dependence again becomes uniformly distributed in the interval $[0,2 \pi[$.

\section{Particle in a sector of angle $\Theta$ with reflecting boundaries}

$$
\begin{array}{r}
P_{D}\left(r, \varphi, t \mid r_{0}, \varphi_{0}, t_{0}\right)=\frac{e^{-\frac{r^{2}+r_{0}^{2}}{4 D\left(t-t_{0}\right)}}}{2 \Theta D\left(t-t_{0}\right)}\left[I_{0}\left(\frac{r r_{0}}{2 D\left(t-t_{0}\right)}\right)+\right. \\
\left.2 \sum_{n=1}^{\infty} \cos \left(n \frac{\pi \varphi}{\Theta}\right) \cos \left(n \frac{\pi \varphi_{0}}{\Theta}\right) I \frac{n \pi}{\phi}\left(\frac{r r_{0}}{2 D t}\right)\right]
\end{array}
$$

where $I_{\omega}$ denotes the modified Bessel function of order $\omega$.

The density for finding the particle at an arbitrary angle $\varphi$ at radius $r$ is then given by

$$
\rho_{r}\left(r, t \mid r_{0}, t_{0}\right)=\frac{r e^{-\frac{r^{2}+r_{0}^{2}}{4 D\left(t-t_{0}\right)}}}{2 D\left(t-t_{0}\right)}\left[I_{0}\left(\frac{r r_{0}}{2 D\left(t-t_{0}\right)}\right)\right] .
$$

As there is no analytic expression for a distribution function of $\rho_{r}$ available, the usage of the inversion method would be very slow, as the integration of $r$ would have to be done numerically. Fortunately, $\rho_{r}$ does not depend on the sector angle $\Theta$, hence the analytically known solution for $\Theta=\pi$ (half-plane) can be used to generate $r$ for all $\Theta$. A distribution function for the angle $\varphi$ under the condition of being at radius $r$ can be derived straightforwardly by integrating the cos-function.

\section{Particle in a sphere of radius $R$ with absorbing boundary conditions}

$$
\begin{aligned}
& P_{D}\left(r, \varphi, \vartheta, t \mid r_{0}, \varphi_{0}, \vartheta_{0}, t_{0}\right)= \\
& \frac{1}{2 \pi R^{2} \sqrt{r r_{0}}} \sum_{n=0}^{\infty}(2 n+1) P_{n}\left(\mu\left(\varphi, \vartheta, \varphi_{0}, \vartheta_{0}\right)\right) \\
& \cdot \sum_{\alpha_{n}} e^{-\alpha_{n}^{2} \frac{D\left(t-t_{0}\right)}{R^{2}}} \frac{J_{n+\frac{1}{2}}\left(\alpha_{n} \frac{r_{0}}{R}\right) J_{n+\frac{1}{2}}\left(\alpha_{n} \frac{r}{R}\right)}{\left[J_{n+\frac{1}{2}}^{\prime}\left(\alpha_{n}\right)\right]^{2}}
\end{aligned}
$$

where $\sum_{\alpha_{n}}$ denotes the infinite sum over all positive zeros $\alpha_{n}$ of the Bessel function $J_{n+\frac{1}{2}}\left(\alpha_{n}\right)=0, P_{n}$ is the n-th Legendre polynomial and $\mu$ is the cosine of the angle between $\mathbf{r}$ and $\mathbf{r}_{0}$.

The density of finding the particle at arbitrary angles $\varphi, \vartheta$ at radius $r$ is then given by

$$
\begin{aligned}
& \rho_{r}\left(r, t \mid r_{0}, t_{0}\right)= \\
& \int_{0}^{2 \pi} d \varphi \int_{0}^{\pi} d \vartheta P_{D}\left(r, \varphi, \vartheta, t \mid r_{0}, \varphi_{0}, \vartheta_{0}, t_{0}\right) \sin (\vartheta) r^{2} \\
& =\frac{2 r^{\frac{3}{2}}}{R^{2} \sqrt{r_{0}}} \sum_{\alpha_{0}} e^{-\alpha_{0}^{2} \frac{D\left(t-t_{0}\right)}{R^{2}}} \frac{J_{\frac{1}{2}}\left(\alpha_{0} \frac{r_{0}}{R}\right) J_{\frac{1}{2}}\left(\alpha_{0} \frac{r}{R}\right)}{\left[J_{\frac{1}{2}}^{\prime}\left(\alpha_{0}\right)\right]^{2}} \\
& =\frac{2 r}{R r_{0}} \sum_{n=1}^{\infty} e^{-n^{2} \pi^{2} \frac{D\left(t-t_{0}\right)}{R^{2}}} \sin \left(\frac{n \pi r_{0}}{R}\right) \sin \left(\frac{n \pi r}{R}\right) .
\end{aligned}
$$

The corresponding distribution function can be derived by integrating the sin-functions:

$$
\begin{aligned}
F_{r}\left(r, t \mid r_{0}, t_{0}\right) & =\frac{2}{R r_{0}} \sum_{n=1}^{\infty} e^{-n^{2} \pi^{2} \frac{D\left(t-t_{0}\right)}{R^{2}}} \sin \left(\frac{n \pi r_{0}}{R}\right) \\
\cdot & {\left[\frac{R^{2}}{n^{2} \pi^{2}} \sin \left(\frac{n \pi r}{R}\right)-\frac{R}{n \pi} r \cos \left(\frac{n \pi r}{R}\right)\right] }
\end{aligned}
$$

Hence, the distribution function belonging to $\rho_{b}^{D}$ is given by

$$
\begin{aligned}
& F_{b}^{D}\left(t \mid r_{0}, t_{0}\right)= \\
& 1-\frac{2 R}{\pi r_{0}} \sum_{n=1}^{\infty} e^{-n^{2} \pi^{2} \frac{D\left(t-t_{0}\right)}{R^{2}}} \sin \left(\frac{n \pi r_{0}}{R}\right) \frac{(-1)^{n+1}}{n},
\end{aligned}
$$

which was used to derive Eq. (15) with the help of l'Hospital's rule $\left(r_{0} \rightarrow 0\right)$.

A distribution function for $\mu \in[-1 ; 1]$ under the condition of being at radius $r$ can be derived straightforwardly by integrating the Legendre polynomials $P_{n}$. Using the sampled $\mu$, the angels $\varphi$ and $\vartheta$ are sampled. 
[1] S. S. Andrews and D. Bray, Stochastic simulation of chemical reactions with spatial resolution and single molecule detail, Phys. Biol. 1, 137 (2004).

[2] J. Elf and M. Ehrenberg, Spontaneous separation of bistable biochemical systems into spatial domains of opposite phases, Syst. Biol. 1, 230 (2004).

[3] D. Fange, O. Berg, P. Sjöberg, J. Elf, Stochastic reactiondiffusion kinetics in the mircroscopic limit, Proc. Nat. Acad. Sci. 107, 19820 (2010).

[4] J. S. van Zon and P. R. ten Wolde, Simulating biochemical networks at the particle level and in time and space: Green's function reaction dynamics, Phys. Rev. Lett. 94, 128103 (2005).

[5] J. S. van Zon and P. R. tenWolde, Green's function reaction dynamics: A particle-based approach for simulating biochemical networks in time and space, J. Chem. Phys. 123, 234910 (2005)

[6] T. Oppelstrup, V. V. Bulatov, G. H. Gilmer, M. H. Kalos, B. Sadigh, First-passage Monte Carlo algorithm: Diffusion without all the hops, Phys. Rev. Lett. 97, 230602 (2006).
[7] T. Oppelstrup, V. V. Bulatov, A. Donev, M. H. Kalos, G. H. Gilmer, B. Sadigh, First-passage kinetic Monte Carlo method, Phys. Rev. E 80, 066701 (2009).

[8] A. Donev, V. V. Bulatov, T. Oppelstrup, G. H. Gilmer, B. Sadigh, M. H. Kalos, A First-Passage Kinetic Monte Carlo algorithm for complex reaction-diffusion systems, J. Comp. Phys. 229, 3214 (2010).

[9] C. Loverdo, O. Benichou, M. Moreau, R. Voituriez, Enhanced reaction kinetics in biological cells, Nature Physics 4, 134 (2008).

[10] C. Loverdo, O. Benichou, M. Moreau, R. Voituriez, Robustness of optimal intermittent search strategies in one, two, and three dimensions, Phys. Rev. E 80, 031146 (2009).

[11] L. Devroye, Non-Uniform Random Variate Generation, Springer, Heidelberg (1986).

[12] H. S. Carslaw, J. C. J'ager, Condction of heat in solids, Oxford University press, USA (1959).

[13] O. Bénichou, C. Loverdo, M. Moreau, R. Voituriez, Intermittent search strategies, Rev. Mod. Phys. 83, 81 (2011). 